\title{
TRAIN TICKETING SYSTEM USING SMARTCARD
}

\author{
Abu Abraham Mathews ${ }^{1}$, Amal Babu $\mathbf{P}^{2}$ \\ ${ }^{I}$ III YEAR, Department of Electronics and Instrumentation Engineering, Karunya University, Tamilnadu, India \\ ${ }^{2}$ III YEAR, Department of Electronics and Instrumentation Engineering, Karunya University, Tamilnadu, India
}

\begin{abstract}
The goal of our project is to attain improved travel information and electronic ticketing using smart cards. The smart cards are similar to that of an ATM, so that they can be recharged and can be reused often. Smart cards are secure portable storage devices used for several applications especially security related ones involving access to the system's database. This looks into current trends in smart card technology and highlights what is likely to happen in the future. The smart card has a microprocessor or memory chip embedded in it that, when coupled with a reader, has the processing power to serve many different applications. The smart cards are user- friendly and so it can be used for Public Transport Networks (PTNs).It can also be noted as a service-oriented architecture. Railways are the important key aspect for the development of the Indian revenue. Many people are in need of train transportation than any other means of transportation because a number of people may travel at the same time. So people may prefer smart cards for the traveling purpose instead of booking the tickets. The existing fare booking system can be replaced by smart cards.
\end{abstract}

Keywords: - Public Transport Networks (PTNs), Synchronous Serial Port (SSP), Power-on Reset(POR) and Power-up Timer $(P W R T)$

\section{INTRODUCTION}

Every man since came into existence, the thrust for knowledge and invention has been an unquenchable process, thanks to which man has giant leaps in technology which couldn't have been ever thought before. One such contribution is the development of microcontroller and smart card.

There are many applications of smart card that are widely being used. This project aims at smart card based ticketing system will carry all modes of ground transportation nationwide including railways. Railway has a valuable role in economic development of each country. In railways the ticketing system in India is done in two ways till now. The first one is that the individual himself goes to the counter and book the ticket i.e., reservation ticket. The second is an alternative provided by the Indian Railways is to use Information Technology so that passengers may use online booking of rail tickets. We may also book tickets through the agents approved by indian railways.

Though the existing method has advantages there are many SERIOUS problems, some are to book a general class ticket, one has to go to the railway station or book from the authorized agents, but this process is quite time consuming and people don't like to stand in queues because in every important junctions and platforms due to arrival of trains there may be a lot of crowd. Due to this reason, people are afraid of losing the train, so some people travel without ticket. This has become the most serious issue for our country's development.
As the Indian Railways is one of the largest organizations in the world, and the country's revenue is mainly dependent on the railways. This may be affected by the above issues so it can be eliminated from our project's proposed work. In future improved assessment of the above technique is that we may debit the amount required to recharge the smart card directly from our personal account. This new smart card payment is efficient for revenue management.

The objective of our project is introducing smart card based ticketing system in railway. So the passenger to get the ticket easily and it can be recharged also. It helps to save time and energy.

\subsection{Overview of Project Work}

A new technique for ticketing system in railway by using smart card. We can see that effective use of technology could enhance its contribution to the development of the nation. A smart card based ticketing system will carry all modes of ground transport nationwide including railways. When it comes to public transit, passenger demands are clear commuters want convenient, affordable and efficient options for travel. Smart card solutions in the form of electronic ticketing promise to deliver on those demands. Smart cards are more convenient, they can be purchased and "reloaded" using automated processes and in some cases at home on the Internet. Because cards can be personalized, they can be cancelled if stolen. They are also more efficient to use: the contact functioning of many cards allows more rapid movement through stations and onto different modes of travel. 


\section{PROPOSED METHOD}

\subsection{Introduction}

The proposed advancement of our project is the introduction of smart cards. The smart cards are a type of ATM cards, by which the passengers may travel from one place to another simply by scratching the card in the driver machine. The more number of driver machine (or) the vending machine placed in every railway station helps to verify the codes and prints the ticket for the passenger [6].

We may also recharge the card at any times and may use often, this is the most advantage of our proposed ticketing system. This is the hottest development in the Indian Railways. The codes can be verified by the vending machine and we may also check the remaining balance also.

\subsection{Advantages}

- Smart cards are small, they fit everybody's pocket.

- Smart cards are very secured, can't be read, copied, manipulated with, counterfeited, and duplicated.

- Smart card protect privacy where needed.

- Smart cards are re-writable and can be recharged thousands of times.

- Smart card can contain digital log with location, date, time, person's stamp to record every transaction.

- Smart card can contain money value in electronic form, kind of small system.

- Smart card can contain weekly pass, monthly pass or simply ticket that expires by certain date.

- Smart card fare system eliminates cash from the payment systems.

- Smart card fare systems remove burden of handling cash by drivers. It reduces crime.

\subsection{Applications}

- Avoiding long Queue

- Easy to get the train

- Save the Time

- It is very much used in Metro Cities

\section{BLOCK DIAGRAM}

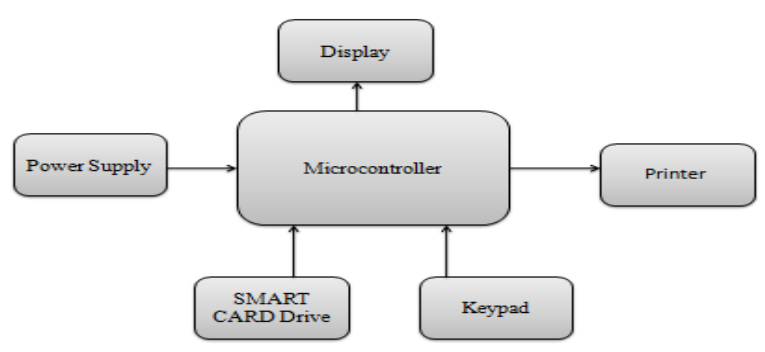

Fig 1 Block diagram
The project module consists of micro controller, power supply unit, keypad, printer, display, smart card drive, etc....

The micro controller (16F877A) is a 40 pins IC where all the details such as train name, ticket details, and locations are preprogrammed in this microcontroller. The $12 \mathrm{v}$ supply can be retarded to $5 \mathrm{v}$ by the power supply unit, the keypad used is $3 * 4$ keypad. The keypad is used to enter the region where a passenger has to move. in addition we use the thermal printer to print the tickets.

\subsection{PIC}

PIC stands for Peripheral Interface Controller coined by Microchip Technology Inc., USA.

\subsubsection{Features}

- PIC is a popular microcontroller Worldwide.

- High performance, cost effective, field programmable embedded control solutions.

- PIC microcontrollers are one of the most secure products of its kind on the market today.

- Wide Applications Area.

- RISC Architecture makes for easy programming.

\subsubsection{Core Features}

- High performance RISC CPU with 35no.of instruction set only.

- All single cycle instructions except for program branches which are two cycle.

- Operating speed: DC-20MHz clock input with DC to $200 \mathrm{~ns}$ instruction cycle.

- Up to $8 \mathrm{~K}$ x14 words of FLASH Program Memory.

- Up to $368 \times 8$ bytes of Data Memory(RAM).

- Up to 256 x 8 bytes of EEPROM Data Memory.

- Interrupt capability(up to 14 sources).

- Direct, indirect and relative addressing modes.

- Power-on Reset(POR) and Power-up Timer(PWRT)

- Oscillator Start-up Timer(OST)and Selectable oscillator options.

- Watching Timer(WDT) with its own on-chip RC oscillator for reliable operation.

- Programmable code protection and Power saving SLEEP mode.

- Low power, high speed CMOS FLASH/EEPROM technology.

- Fully static design with In-Circuit Serial Programming (ICSP)via two pins.

\subsubsection{Peripheral Features}

- Timer0: 8-bit timer/counter with 8-bit prescaler

- Timer1: 16-bit timer/counter with prescaler, can be incremented during SLEEP via external crystal/clock. 
- Timer2: 8-bit timer/counter with 8-bit period register , prescaler and postscaler.

- Synchronous Serial Port(SSP) with SPI.(Master mode)and 12C.(Master/Slave)

- Universal Synchronous Receiver Transmitter (USART/SCI) with 9-bit address detection.

- Parallel Slave Port(PSP)8-bits wide, with external RD,WR and CS controls

- Brown -out detection circuitry for Brown-out Reset (BOR).

\subsubsection{PIC Family}

PIC family of microcontrollers is built on Hardware architecture, in which program code and date are stored in separate memory locations. The CPU executes each instruction during the cycle following its fetch, pipelining instruction fetches and instruction execution to achieve the execution of one instruction every cycle. This sequence of execution is broken and an additional cycle is introduced when executing GOTO instruction. The PIC controllers are available in different types of packages. We will focus on PIC16F877A controller for our explanation.

Speed: PIC executes most of its instruction in $0.2 \mu \mathrm{s}$ or five instruction per $\mu \mathrm{s}$.

Instruction Set Simplicity: The instruction set consists of just 35 instructions.

Instruction of Operational Features: Power on reset and brown-out protection ensure that the chip operates only when the supply voltage is within the specification. A watchdog timer resets if the chip ever malfunctions and deviates from its normal operation.

- Programmable Timer Options

- Interrupt Control

- Powerful Output Pin Circuit

- I/O port Expansion

- Serial Programming via two pins

- EPROM and RWM options

\subsection{Smart Card}

Smart cards are secure portable storage devices used for several applications especially security related ones involving access to system's database either online or offline. For the Future of smart card to be bright, it is important to look into several aspects and factors especially those resulted due to the rapid advancement in information and communication technology. This is looks into current trends in smart card technology and highlights what is likely to happen in the future. The other aspects in order to identify the core concepts that are of interest to smart card developers and researchers. More emphasis is given to four key characteristics of smart cards: portability, security, open platform, and memory management, as they are believed to be at the heart of many smart card applications.
Smart card is one of the greatest achievements in the world of information technology. Similar in size to today's plastic payment card, the smart card has a microprocessor or memory chip embedded in it that, when coupled with a reader, has the processing power to serve many different applications. As an access-control device, smart cards can be used to access server remotely over the Internet and they can make personal and business data available only to the appropriate users. Smart cards provide data portability, security, convenience.

Smart cards help businesses evolve and expand their products and services in a changing global marketplace. The scope of uses for a smart card has expanded each year to include applications in a variety of markets and disciplines. In recent years, the information age has introduced an array of security and privacy issues that have called for advanced smart card security applications.[7]

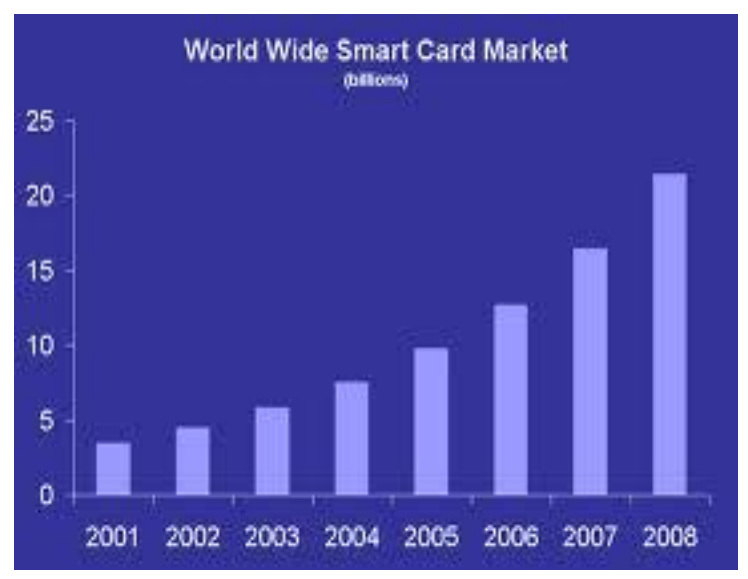

Chart 1 Smart card Market

\subsubsection{Smart Card Reader}

A smart card reader is an electronic device that reads smart cards and can be found in the following forms

- Some keyboards have a built-in card reader.

- External devices and internal drive bay card reader devices exist for personal computers (PC).

- Some laptop models contain a built-in smart card reader and/or utilize flash upgradeable firmware. 


\subsection{Flow Chart}

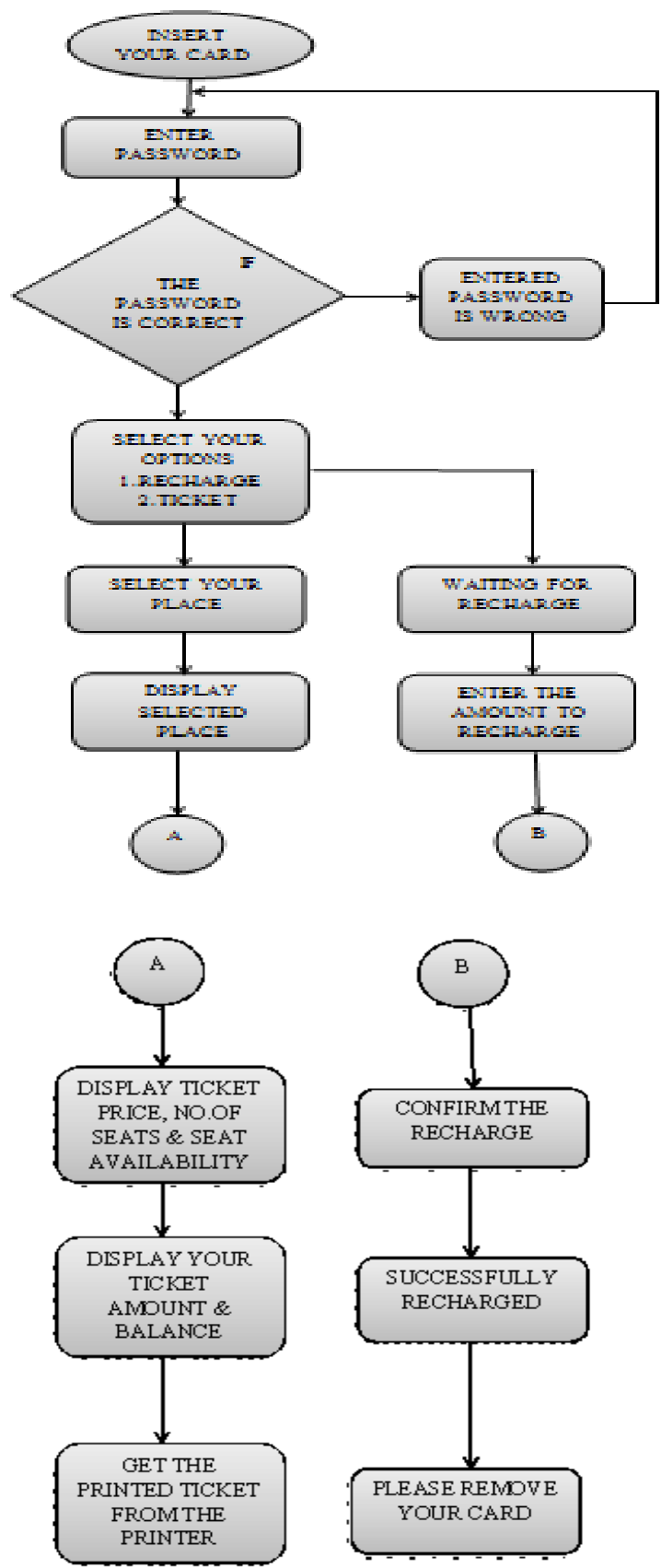

Fig 2 Flow chart

\subsubsection{Algorithm}

STEP 1 : Insert the SMARD CARD and enter the secret password.

STEP 2 : Check whether the entered password is correct or not .If the password is correct it will go to the next step. If it is wrong it will go to the previous step.
STEP 3 : To select our option from these two options 1.Recharge, 2.Ticket. If we select option 2, the following steps to be proceed.

STEP 4 : Select our destination place and number of tickets. STEP 5 : Then the following details will be displayed ticket price, number of seats, seat availability.

STEP 6 : The printed ticket will be collected from the printer else if we select option 1, the following steps to be proceed.

STEP 7 : Enter the amount to recharge

STEP 8 : Confirm the amount to recharge or cancel

STEP 9 : Then the following details will be displayed successfully recharged and remove your card.

\subsection{Software}

- PIC COMPILER

- Proteus 7.6

\section{SIMULATION RESULTS}

As soon as the process begins the smart card is inserted and our system requires the cards unique password

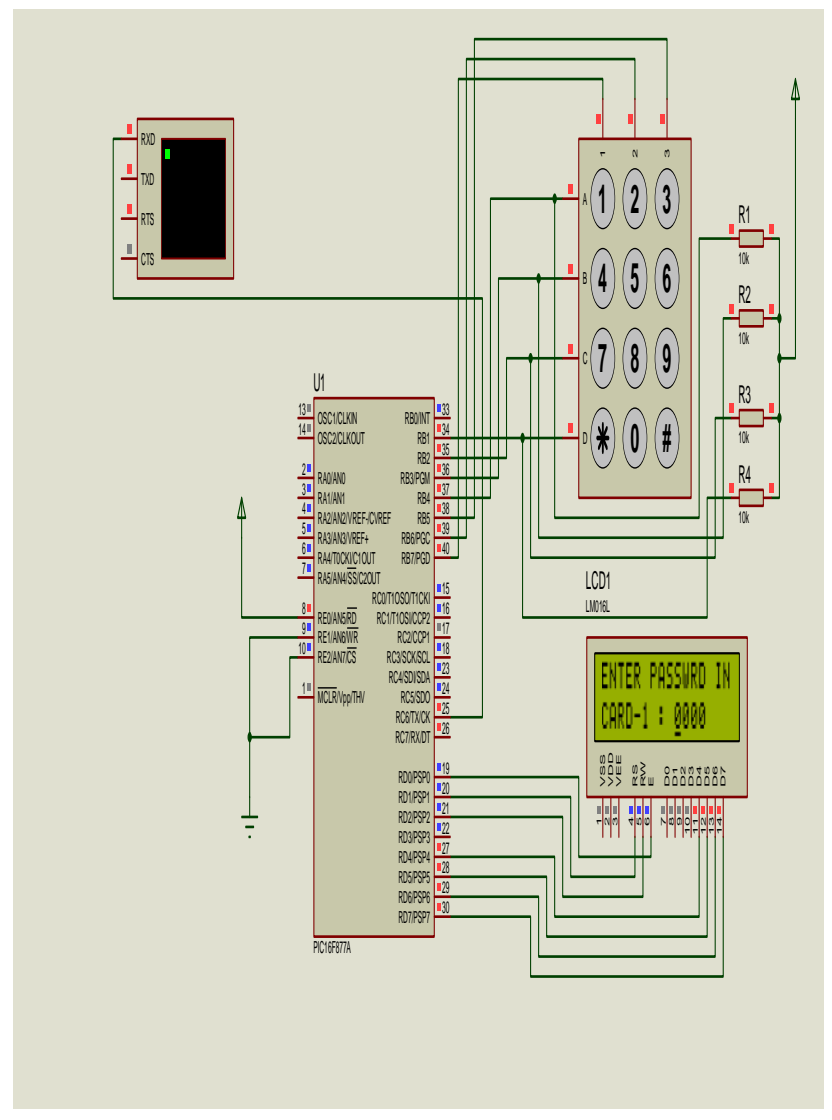

Fig 3 Entering password 
Now, our system provides the smart card user with two options 1.Recharge and 2.Tickets

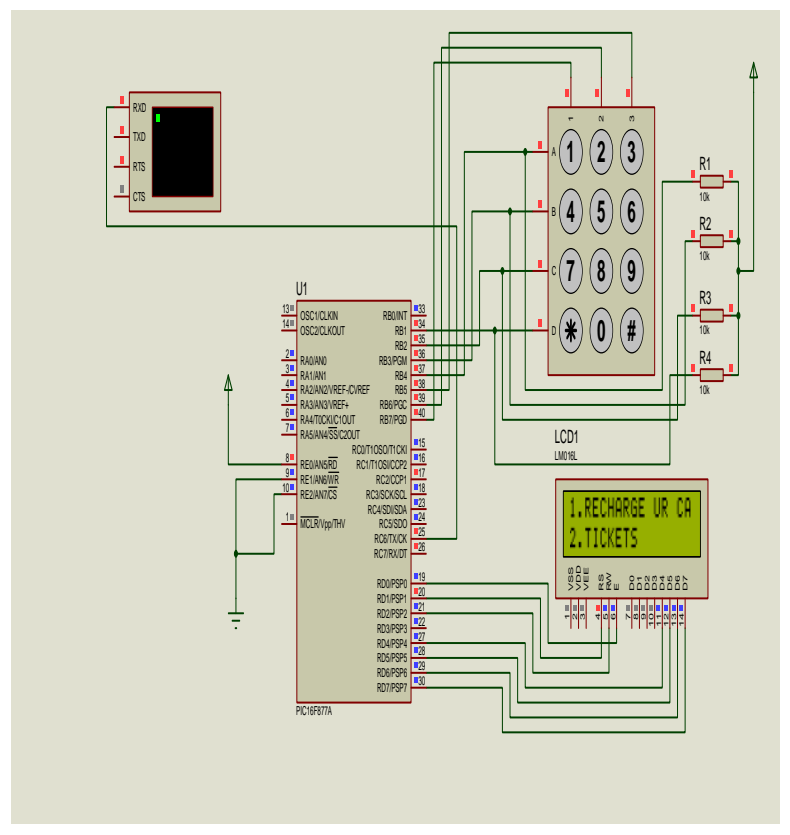

Fig 4 Selection of options

After then our system enables the smart card user to select the place for locomotion

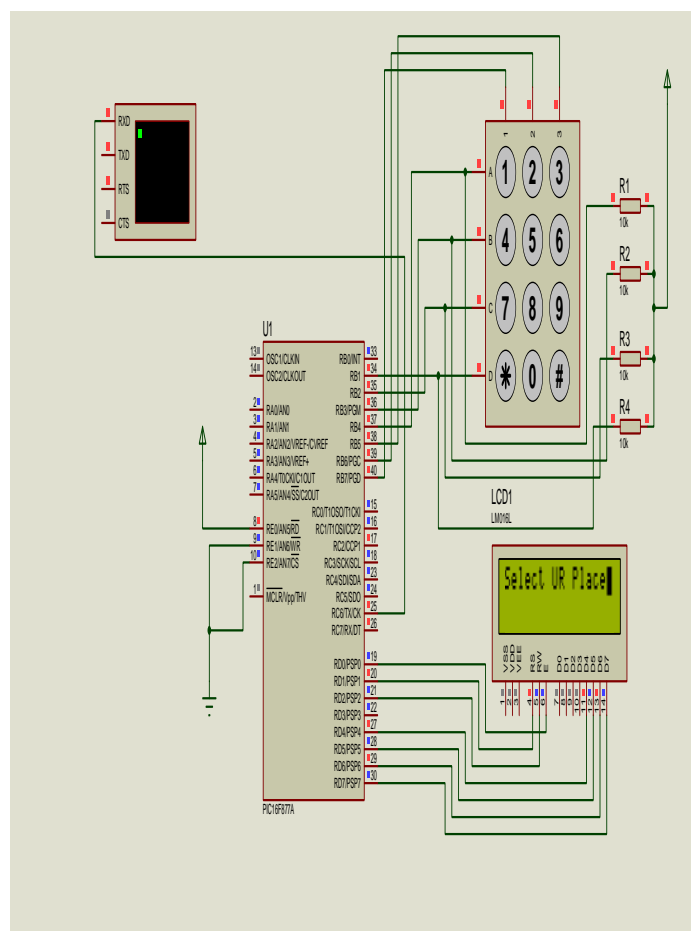

Fig 5 Selection of place
After the place is selected our system checks for seat availability.

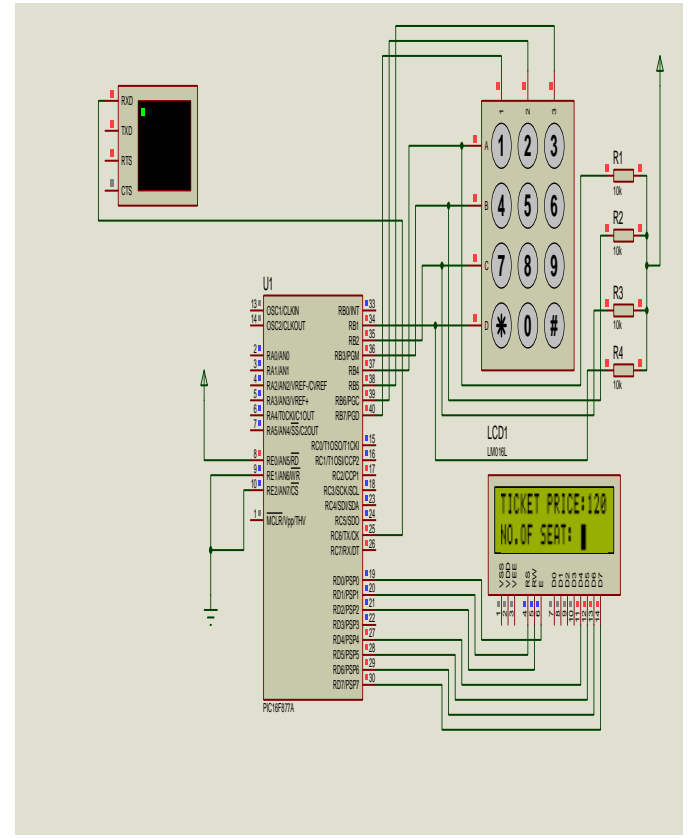

Fig 6 Displaying ticket price

If the seat is available our displays the seat is available and then the process continues

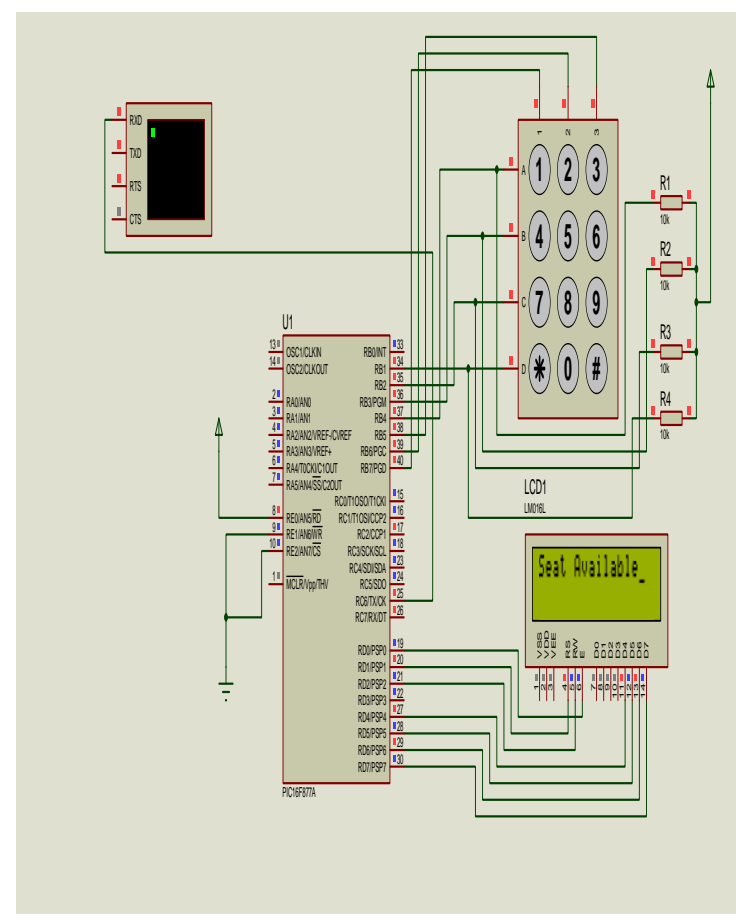

Fig 7 Seat availability 
Finally the ticket is generated, and the remaining balance in our smart card is also displayed

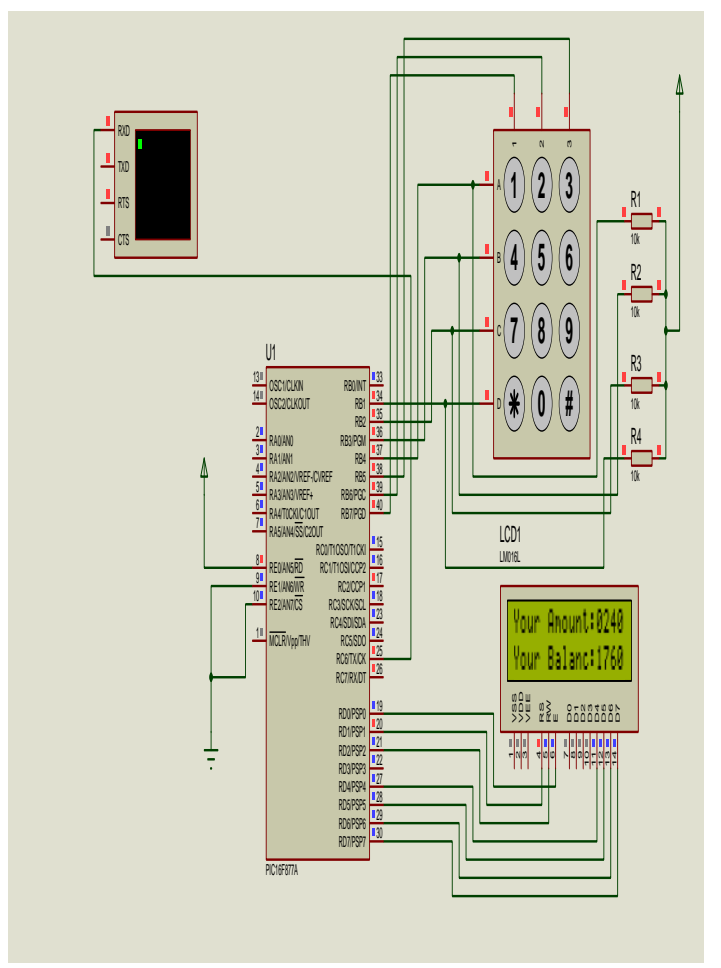

Fig 8 Balance checking

\section{CONCLUSIONS}

The goal of our project is attained the aim, using this system the passenger can easily get the train ticket without wasting their time to wait in a long queue. This system is mainly used to get general tickets and the card can be recharge by monthly basis. Newly, Smart card technology are being used in a number of ways around the world on the other hand, security has become significant in information technology, especially in those application involving data sharing and transactions. These features make it a real time project with a good commercial and social value.

\section{FUTURE DEVELOPMENT}

- The cost of the project can be effectively reduced by mass production.

- By utilizing VLSI technology the size of the device can be more compact.

- In future we can implement ticket reservation and cancelling in this system.

- In future we can implement the recharge system by directly transferring the amount from our bank balance. SMS alert System Can also implement in future.

\section{REFERENCES}

[1] Akintunde Ibitayoakinwande, Ioannis Kymissisand John Sarik, 'A Laboratory Based Course in Display Technology', IEEE Trans. Educ., vol. 54, no. 2, May.2011.

[2] ApostolosFournaris, George Kostopoulos, George Selimis, and Odysseas Koufopavlou , 'Software and Hardware Issues in Smart Card Technology', IEEE Commun., Vol.11.no.3,2009.

[3] Amit Kumar Gupta and PriyankaAhlawat Mann , 'Railway Train Ticket Generation A Business Application for Indian Railways', An international Journal of Computer Applications, vol.22, no.7, May.2011.

[4] K.Mayes and K.Markantonakis , Smart Cards, Tokens, Security \& Application, Springer, Jan 2008. 